\title{
Expérience auditive et mémoire phonique dans les entretiens avec des spectateurs de théâtre
}

Aural experience and vocal memory in interviewing members of a theatre audience

\section{Hélène Bouvier}

\section{CpenEdition}

\section{Journals}

Édition électronique

URL : http://journals.openedition.org/rsl/1200

DOI : $10.4000 /$ rsl. 1200

ISSN : 2271-6246

Éditeur

Éditions Rue d'Ulm

Référence électronique

Hélène Bouvier, «Expérience auditive et mémoire phonique dans les entretiens avec des spectateurs de théâtre », Revue Sciences/Lettres [En ligne], 5 | 2017, mis en ligne le 02 octobre 2017, consulté le 22 avril 2019. URL : http://journals.openedition.org/rsl/1200; DOI : 10.4000/rsl.1200

Ce document a été généré automatiquement le 22 avril 2019.

(c) Revue Sciences/Lettres 


\title{
Expérience auditive et mémoire phonique dans les entretiens avec des spectateurs de théâtre
}

\author{
Aural experience and vocal memory in interviewing members of a theatre \\ audience
}

Hélène Bouvier

1 «Il s'agit d'explorer la mémoire théâtrale des spectateurs pour vérifier si la prééminence du visuel sur le sonore et le verbal dans le discours sur le théâtre moderne et contemporain est ou non validée par les souvenirs et les marques que ce théâtre a laissés en eux, dans la longue durée »: telle est la proposition de recherche de départ pour le volet « Mémoire théâtrale et archives sonores. Enquête de terrain » du projet ANR ÉCHO [ÉCrire l'Histoire de l'Oral. L'émergence d'une oralité et d'une auralité modernes. Mouvements du phonique dans l'image scénique (1950-2000)] dirigé par MarieMadeleine Mervant-Roux. Ainsi se trouvent résumées la recherche menée jusqu'à présent et les questions qu'elle a aussitôt soulevées concernant la mémoire courte, la mémoire longue, la mémoire réactivée, retravaillée, l'empilement, l'écrasement, l'effacement par le temps ou la redondance, jusqu'à notre interrogation finale : où se loge le sonore dans ce processus complexe, sensible et cognitif?

2 Concrètement, dans le cadre du projet ÉCHO qui se concentre sur la période allant de l'après-guerre à la fin des années 1990, et sur trois lieux principaux, la grande salle du TNP, le théâtre de l'Athénée et la Cour d'honneur d'Avignon, il s'agit de solliciter par un entretien la mémoire théâtrale d'un spectateur, ce qui lui revient sous une forme ou une autre (sonore, visuelle, verbale, sociale ou affective).

Que reste-t-il dans nos cœurs et dans nos esprits des spectacles de théâtre que nous avons vécus? De quoi nous souvenons-nous lorsque nous savons avoir gardé la trace d'une représentation théâtrale ? Comment cet évènement reste-t-il vivant dans notre mémoire ${ }^{1}$ ? 


\section{Le terrain des entretiens}

3 Vingt-quatre entretiens avec vingt-et-une personnes différentes, amateurs ou professionnels de théâtre ont été réalisés, d'abord à deux (avec Pascale Caemerbeke ou avec Leonel Martins Carneiro, qui ont pris une part active au démarrage de ce volet du projet ÉCHO et y contribuent maintenant par des voies conjointes), puis seule pour les trois quarts du total. Ces personnes ont été contactées par le bouche-à-oreille ou ont répondu spontanément à un appel à témoignage distribué une fois à la sortie du Théâtre national de Chaillot et une fois à la sortie du Théâtre de l'Athénée ${ }^{2}$ et publié dans une version abrégée parue dans La Terrasse ( $n^{\circ} 227$, décembre 2014).

\section{Méthode}

4 La difficulté méthodologique de fond, indiquée dès la définition du projet, est celle liée à la diversité constitutive de tout public, surtout si l'on en sollicite les mémoires individuelles, par des témoignages écrits et/ou oraux. Un autre élément important est celui de l'âge des personnes sollicitées. En effet, étant donné la période sélectionnée, ces spectateurs ont déjà l'expérience intime du travail de la mémoire dans tous les domaines de la vie, et savent de première main ce qu'il représente de tri, de choix et de fixation sélective, bien au-delà du souvenir d'une perception globale et passive, donnée une fois pour toutes, et indissociable du processus parfois douloureux de l'oubli. Cette situation de conscience partagée du statut de la mémoire s'est avérée être un atout pour cette recherche. La durée des entretiens varie entre une heure et trois heures, avec une moyenne de deux heures, au domicile de la personne ou dans un café. Ils ont tous été enregistrés en audio, les tout premiers aussi en vidéo, et une partie des entretiens a été transcrite. Certains échanges se sont poursuivis dans un second entretien, à une date rapprochée (quelques jours plus tard), quand le temps avait manifestement manqué la première fois. À l'issue de chaque rencontre, la question d'un éventuel nouvel entretien a été posée, dans le but de préciser les spectacles, ou les traces mémorielles qui nous intéressent plus particulièrement dans le registre sonore, ou encore pour saisir comment la mémoire aura continué à évoluer et à travailler entretemps, révélant peut-être des aspects non anticipés. À une seule exception près, tous les interlocuteurs ont donné une réponse positive, et même enthousiaste, à cette demande.

\section{Les questions abordées lors des entretiens}

5 Les entretiens ont tous suivi le même canevas, base et aide-mémoire du chercheur, comme autant de pistes proposées à l'interlocuteur, qu'il a saisies et développées à chaque fois différemment. Ces questions sont données dans l'ordre le plus fréquemment suivi :

- le rappel de la période et des trois lieux de référence comme point de départ de la mémoire théâtrale sollicitée ;

- un premier souvenir de théâtre, ou le souvenir du premier spectacle de théâtre, ou encore le souvenir le plus marquant, le plus fort, le plus pérenne, même en dehors des trois lieux de référence du projet ; 
- le rappel d'un jeu, d'une voix de comédien, d'un corps, d'un son, d'un mouvement, d'une présence ;

- le rappel de répliques, de textes, de scènes ;

- celui d'une scénographie, d'une mise en sons ou d'une création sonore particulière ;

- les impressions générales, l'ambiance d'un spectacle, d'une salle, d'un public ;

- les incidents, les surprises ;

- le contexte social de la sortie au théâtre: seul ou accompagné (famille, amis, conjoint, camarades de classe, sortie scolaire avec un professeur ou avec des élèves) ;

- le souvenir du même spectacle vu plusieurs fois ;

- ce qui reste habituellement en mémoire d'un spectacle ;

- la motivation pour aller voir ce spectacle : auteur, salle, pièce, acteurs, amis, etc. ;

- comment mettre en relation l'expérience vécue au théâtre avec celle de la radio, des concerts, de la danse, de l'opéra, du cinéma, de la télévision, des disques et cassettes, de la lecture de théâtre?

- qu'est-ce qu'on oublie?

- si vous en aviez la possibilité, aimeriez-vous réécouter les spectacles que vous avez évoqués? Si oui, lesquels?

- seriez-vous d'accord pour poursuive avec un autre entretien, dans le cadre de cette recherche sur la mémoire théâtrale?

6 Pour les professionnels du théâtre, les professeurs de lettres qui emmènent leurs classes au théâtre, les professionnels de la culture (radio, etc.), s'ajoutent quelques questions complémentaires: ce que vous allez voir, et dans quel but; ce que cela change d'être (comédien, metteur en scène, auteur...) en tant que spectateur et ce que vous pensez d'une dissociation éventuelle entre spectateur et professionnel du théâtre.

\section{Un premier bilan des récurrences}

Il s'agit clairement, lors d'un premier entretien avec un spectateur, d'une mise en route de la mémoire, mais elle s'amorce en réalité avant la rencontre, dès la prise de rendezvous ou dès la lecture de l'appel à témoignage. Si l'interlocuteur ne proposait pas de luimême une première accroche de mémoire théâtrale, lancer cette quête de souvenirs avec " ce qui vient à l'esprit en premier ", ou bien «le plus marquant », ou encore «le premier spectacle de théâtre » a semblé le plus naturel, et s'est révélé efficace. À la réflexion, cela revient à emprunter trois voies d'approche différentes: l'appel rapide ou surgissement spontané, l'empreinte émotionnelle et la source chronologique biographique. Ces différents registres correspondent aux expériences relatées au cours des entretiens et sont confirmés par les points récurrents relevés dans ces échanges. Ainsi l'importance de l'émotion vécue au moment du spectacle, inscrite dans la mémoire, réactivée pendant l'entretien, et parfois même analysée par l'interlocuteur au cours de l'échange, est manifeste. De même, le souvenir du silence d'une salle, de son attention et de sa captation par la scène, reste prégnant. La qualité de la diction d'un acteur laisse une empreinte sémantique et sonore encore vivace, souvent associée à la sensation de sa présence sur scène. Un spectacle qui laisse une impression de partage, de don, de rencontre entre comédiens et public, reste comme une expérience forte, ressentie comme étant à la fois individuelle et collective. Les conditions de la sortie au théâtre (motivation, contexte, et parfois, place dans la salle) jouent un rôle déterminant dans le processus de remémoration. Les premières fois, mais surtout l'association à l'enfance, à l'adolescence ou au tout début de l'âge adulte marquent généralement la mémoire de manière 
indélébile. Enfin, la reconnaissance de l'action sur soi d'un spectacle de théâtre considéré comme marquant, à travers des suites professionnelles, un «accompagnement » dans la vie quotidienne, dans certains moments de la vie, ou même la transformation ou la maturation de soi-même, est également un élément récurrent ${ }^{3}$. À ce stade intermédiaire de l'analyse, les éléments recueillis ne révèlent pas un ordonnancement discernable, tantôt détails microscopiques qui pointent, tantôt souvenirs fulgurants qui colorent la mémoire globale ou le son d'un spectacle, choc indélébile ou impression difficile à isoler et à se remémorer.

8 Certaines opinions ne sont émises que par les spectateurs amateurs de théâtre, non reprises par les créateurs ou professionnels de la culture, comme la nécessité de bien entendre pour comprendre, de bien entendre le texte, la nécessité des emplois et la gêne éprouvée devant les comédiens qui "dépassent " leur personnage, la gêne pour les accents en langue étrangère ou la contrainte des sur-titrages. La plupart des interlocuteurs, quelle que soit leur profession, ont en commun l'amour du texte, de la lecture, de l'apprentissage par cœur, et mettent en avant, pour expliquer leur passion du théâtre, leur goût pour l'expression verbale, sans effectuer de rupture entre écrit et oral.

\section{Pistes d'analyse}

\section{Les éléments de l'expérience sonore initiale}

Certains spectateurs s'attendaient à être sollicités sur leur mémoire sonore, d'autres non, certains s'y étaient préparés, d'autres pas. L'intitulé complet du projet ANR ÉCHO, le texte de l'appel à témoignage, et le premier contact (souvent) téléphonique ou par personne interposée, ont induit des attentes différentes, également liées à l'histoire et à l'individualité de chacun, bien sûr. Dans tous les cas, comme mentionné plus haut, le moteur de la mémoire dirigée vers l'appel s'est mis en route dès que l'interlocuteur y a été exposé, bien avant que l'entretien n'ait effectivement lieu.

Les éléments de l'expérience sonore initiale sont nombreux. Ils regroupent les voix des comédiens (et leur diction, leur timbre et leur rythme caractéristiques), les bruits de salle (annonces, appels, sonneries, réactions du public, bruits de portes et de sièges) et bruits de scène (décors, pas, rideaux), la musique (jouée sur scène ou préenregistrée), les chansons, l'ambiance sonore générale du lieu de spectacle (et la différence notable entre salle fermée et plein air).

11 Ces éléments sont rappelés dans l'effort de mémoire, ou surnagent sans effort manifeste. Ils sont puisés au milieu d'autres souvenirs, ou énoncés de fil en aiguille, ou encore donnés d'emblée sans demande explicite portant spécifiquement sur les éléments sonores. Ils sont souvent mis en relation avec les autres registres de la mémoire multisensorielle du théâtre. Comme ce qui nous intéresse est l'expérience sonore au sein d'une expérience plus large, celle du théâtre, les béquilles sont nombreuses qui s'offrent à ce travail. En effet, la mémoire tente de trouver les mots qui paraissent les plus fidèles à l'expérience initiale telle qu'on en a une trace, telle qu'on l'a gravée, ou en luttant contre l'oubli dans lequel elle est tombée. Cet effort de remémoration entraîne à analyser et à comparer, faisant avancer la récupération d'éléments que l'on pensait oubliés, mais aussi produisant des associations ou des appréciations différentes, et aboutissant parfois à des surprises, des découvertes à posteriori ou des interrogations qui surgissent pour la première fois au moment de l'entretien. Ainsi, l'évocation d'un comédien dans un 
personnage, de son allure, de son costume ou de sa silhouette, peut donner accès au souvenir de sa voix, et même à un essai de caractérisation de cette voix particulière. Ou bien encore, les trompettes d'appel du public de la grande salle du TNP de la période Vilar, dont presque tous les spectateurs se souviennent, sont tantôt associées à l'impression de solennité et de convocation irrésistible qu'elles provoquaient alors, tantôt identifiées d'emblée comme la marque sonore indélébile de cette période, tantôt se révèlent être un fil conducteur qui transporte l'interlocuteur mentalement dans la salle, parfois jusqu'à sa place, et au cœur des spectacles qu'il y a vus. Cette conjonction de mémoire sonore et spatiale aide alors à dévider la pelote de souvenirs plus complexes. C'est donc une véritable composition orale qui est réalisée pour capter et livrer la mémoire ainsi retrouvée.

\section{Quête verbale et quête des souvenirs de théâtre}

Dans l'exercice de l'entretien, la mémoire théâtrale est donc racontée. En parlant, l'interlocuteur donne une forme (verbale) à une mémoire qui ne l'est pas (sauf ponctuellement pour certaines tirades et pour des professionnels ou passionnés de théâtre qui ont acquis certains automatismes de mémorisation du répertoire). La difficulté consiste à aller chercher des souvenirs, à les ranimer et à les exprimer par les mots. L'enquêteur tient aussi une part dans cette "formulation " ou " mise en forme ", tiraillé entre la nécessité d'engager cette dynamique de remémoration et l'exigence d'un interventionnisme minimal, dans cet entre-deux du témoignage et de la conversation qu'occupe un entretien de ce type.

Il semble bien que la quête verbale, qui conditionne la transmission d'une expérience personnelle à autrui, soit menée de manière synchronique avec la quête des souvenirs de théâtre, et remplisse deux fonctions, celle de la remémoration et celle de la communication, dans un double mouvement : vers l'intérieur, rendant accessible ce qui est en soi et parfois dans l'oubli (les souvenirs), tout en le formulant vers l'extérieur, pour l'enquêteur qui écoute et recueille, mais aussi pour soi, de manière explicite, et audible.

Très souvent, l'interlocuteur en train de se remémorer est aussi entraîné à «jouer » le souvenir qu'il rappelle à lui, comme pour mieux le faire surgir et le partager, traversant autant le champ des mots et de la parole que celui de l'expression du visage, des gestes des mains, des postures, s'approchant finalement du jeu théâtral. Dans cet effort, l'expérience initiale est à la fois ressaisie par l'action, par mimétisme, et réinterprétée.

Quand la personne est encouragée à expliquer une sensation, un souvenir, à transmettre à son interlocuteur un élément inconnu de lui, l'échange et le résultat sont intéressants et gratifiants pour les deux, observateurs conjoints de cette dynamique de la remémoration. Tous les témoins disent en fin d'entretien être surpris d'avoir pu retrouver, et parfois ranimer de manière sensorielle (à travers un air ou une chanson), des souvenirs parfois très anciens qu'ils pensaient tombés dans l'oubli. Ils s'étonnent d'avoir pu rappeler une grande partie de leur expérience théâtrale, ce qui provoque une sensation de reprise de possession d'un trésor enfoui, mais dans une captation nouvelle, que l'exercice de l'entretien reformule et présente sous un jour neuf. Il s'agit bien d'une construction, à la fois analytique et synthétique, avec l'acceptation de trous et d'erreurs, mais qui est rendue intelligible et transmissible grâce à la mise en mots. Et c'est ensemble que l'on constate finalement combien les moteurs qui ont livré cette mémoire appartiennent 
autant au registre sensible (affectif et sensoriel) qu'au registre purement logique ou intellectuel.

\section{Spécificités de la mémoire phonique}

Dans cet exercice de remémoration de l'expérience théâtrale, la quête des souvenirs sonores et leur mise en mots occupent une place très particulière : comment dire ce qu'on a entendu, dire le son perçu et ressenti, et le son qui ne résonne plus? La question de la verbalisation de l'expérience sonore se combine en effet avec celle de la nature même de la mémoire phonique. L'expérience classique de l'enregistrement d'un repas montre combien l'oreille et le cerveau humains filtrent et organisent naturellement les sons au moment même de leur perception (par exemple, en adoucissant les bruits de fourchettes et de vaisselle pour privilégier d'autres sons plus significatifs, comme les voix ou la musique), nous laissant une impression qui n'est pas seulement acoustique, dont on peut comparer l'écart avec l'enregistrement par le micro qui a tout capté indistinctement. Ainsi, le souvenir d'une voix de comédien est-il le souvenir d'un résultat, qui est déjà analyse du son selon les filtres individuels que représentent les références personnelles, les goûts et les apprentissages, etc.; il peut perdurer ou varier selon le moment ou le contexte de son rappel.

Plusieurs interlocuteurs disent qu'ils ont encore dans l'oreille le son d'une voix, d'une réplique, d'un dialogue, et certains ressentent encore en l'évoquant le « frisson » qu'une voix particulière leur a procuré (comme Gérard Philipe dans Lorenzaccio, ou Germaine Montero dans Mère Courage, par exemple). Dans ce cas, la mémoire phonique semble combiner deux éléments : la perception d'un son et l'effet qu'il produit sur soi. Le rappel réactive la sensation acoustique de cette voix et l'émotion qu'elle a provoquée. Dans tous les cas, les interlocuteurs sont unanimes sur l'importance de la voix des comédiens, véritable sémantique phonique nécessaire au théâtre et à son impression sur le spectateur.

En plus de la voix parlée, les airs de musique et les chansons « courent ensuite dans la tête ", souvent bien après les spectacles, et certains témoins peuvent les chanter ou les fredonner jusqu'à aujourd'hui (comme "La chanson de l'étoile» dans Les Caprices de Marianne, sur le poème "L'étoile du berger» d'Alfred de Musset). Cette mémoire phonique a parfois été entretenue grâce aux disques de pièces enregistrées, d'extraits ou de chansons tirées des pièces, mais ce n'est pas toujours le cas.

19 Au cours des entretiens, le son est donc rappelé soit comme élément d'un ensemble, soit comme un élément autonome. Dans le premier cas, la mémoire phonique reste enchâssée dans les autres registres sensoriels, et c'est par la recherche des mots qui paraissent les plus fidèles, les plus justes, souvent par association ou par métaphore, que l'on tente d'accéder à l'expérience auditive initiale. Dans l'autre cas, la mémoire phonique se reconstruit dans la production phonatoire de celui qui peut fredonner un air, chanter une mélodie, avec ou sans les paroles, retrouver une tirade, imiter une voix de comédien. L'expérience initiale est alors dépassée, et irrigue les autres registres des souvenirs.

\section{Quels supports pour le recueil et l'analyse?}

Mener des entretiens sur la mémoire théâtrale, et plus particulièrement son volet sonore, revient à adopter le registre oral à la fois comme méthode, comme objet de recherche et 
comme matière recueillie. Du début à la fin de son travail, le chercheur est aux prises avec les sons, les voix, les paroles, les siens comme ceux de ses interlocuteurs et des spectacles de théâtre qui sont évoqués ou écoutés en archives. Cette situation particulière amène plusieurs réflexions sur le médium choisi pour le recueil et l'analyse.

21 La première porte sur la mémoire que le chercheur lui-même garde des entretiens qu'il a menés et qu'il tente d'analyser. Quand j'ai écouté les enregistrements audio, j'ai été frappée par la voix de mes interlocuteurs, ses inflexions, ses nuances, comme si je ne l'avais pas perçue avec autant d'acuité sur le moment, in vivo. Cette constatation en amène une autre : en regardant son interlocuteur pour mieux comprendre et suivre sa quête de mémoire, quand on interagit et qu'on mène l'entretien, on écoute moins. Être dans l'action et dans une légère anticipation par rapport à ce qui est dit, entraîne une sorte de dissociation dans l'écoute, à la manière du musicien qui est à la fois dans et devant le son qu'il produit, dans un immédiat sans cesse étiré. À l'écoute des enregistrements, c'est la voix, unique, sa matérialité, qui « saute aux oreilles », comme si elle s'était évanouie dans la fixation du souvenir de l'entretien. L'enregistrement audio, - même si l'on concède qu'une captation ne peut saisir le son dans son exhaustivité et suit des critères sélectifs -, lui rend une palette sonore qu'elle avait perdue dans le processus de l'écoute inévitablement filtrée du chercheur. Ainsi ranimé dans une dimension sonore que l'entretien avait oblitérée, le cheminement de la quête mémorielle du spectateur ressort de manière plus concrète et sensible, servant l'effort d'interprétation du chercheur. Et l'on peut constater combien l'expressivité de la voix comme son, qu'elle soit médium de la parole ou mise en voix d'un texte écrit, révèle finalement son efficacité dans toutes les situations, non seulement au théâtre mais également dans les entretiens, et probablement tout échange conversationnel.

Une deuxième réflexion touche la question du choix entre enregistrement audio et vidéo, qui s'est posé au début de la recherche. À titre d'essai, les premiers entretiens ont aussi donné lieu à quelques séquences enregistrées sur vidéo, en plus de l'audio au long cours. Une part indicible de la mémoire théâtrale est peut-être captée par l'expression du visage, un geste, un regard, mais pose la question de son interprétation et de son analyse par le chercheur. Et on peut se demander si l'on dépasse la seule empathie envers celui qu'on regarde, et le plaisir du partage de cette émotion, et comment ce support visuel peut faire avancer l'analyse. C'est pourquoi, seul l'enregistrement audio, déjà riche de défis, comme on vient de le voir, a été finalement retenu comme support, également pour son adéquation avec le registre sonore de la démarche globale.

La dernière réflexion porte sur l'articulation entre transcription écrite et enregistrement audio. Partie de l'évidence de la nécessité d'une transcription verbale complète des entretiens, l'expérience de la réécoute des enregistrements a nuancé ce postulat de départ. Quel que soit son raffinement, une transcription écrite fixe sur le papier les paroles échangées comme autant de papillons épinglés, alors que l'enregistrement leur laisse l'intensité, le timbre et la durée, le souffle. Si le recours aux transcriptions offre un raccourci pratique pour l'analyse des thèmes et du vocabulaire, il semble peu adapté à l'évaluation du rythme de l'élocution et de la remémoration, de la dynamique du dialogue, du déroulement et du dépliement de la mémoire. Le retour à l'audio suppose par contre un travail dans une durée incompressible, et même augmentée par les nécessaires reprises. Comme en musique, on sait qu'on ne peut faire l'économie du déroulement du temps pour saisir cette expérience et l'étudier ${ }^{4}$. On peut considérer que ces deux systèmes, l'analyse des transcriptions écrites et l'écoute attentive des enregistrements 
audio, correspondent à des besoins différents, et se complètent en partie. Il me semble pourtant adéquat, pour une recherche sur la matière et la mémoire sonores, de rester le plus possible dans leur dimension temporelle et leur registre sensoriel.

\section{Le cas de la « période Vilar»}

Pour l'ensemble des spectacles montés par Jean Vilar, les spectateurs décrivent une expérience phonique (voix et diction des acteurs, chansons) qui s'insère dans un ensemble sonore (musique de Maurice Jarre, acoustique de la salle et de la scène, réactions d'un public familial et attentif, annonce avec sonnerie de trompettes), lui-même en correspondance avec les autres éléments, non sonores, tels que le choix des costumes ou des décors, des lumières. Leur mémoire est explicitement associée à un lieu, la grande salle du TNP, même si certains spectateurs ont aussi vu la troupe au Festival d'Avignon. Enfin, un point commun, exprimé de manière insistante, est le souvenir d'un public " accueilli », attendu, et qui rend la réciproque à la troupe. Au-delà de la découverte, du plaisir ou de l'enthousiasme, il s'agit toujours d'une rencontre, qui devient pour beaucoup un rendez-vous.

À partir de l'écoute des archives audio de cette "période Vilar ", et des entretiens qui en ont découlé, on peut se demander comment la mémoire personnelle se place au sein d'une mémoire collective, qu'elle soit simplement générationnelle, sociale, culturelle ou professionnelle. En effet, à l'issue des entretiens, les spectateurs indiquent souvent qu'ils viennent pour la première fois d'embrasser d'un coup une période, un moment, qualifié parfois d'« historique ", avec la nette impression de l'avoir en partage avec tous ceux qui l'ont vécu. Il semble que cette impression ne soit pas aussi claire pour les périodes qui ont suivi, indiquant peut-être qu'une conjonction particulière des domaines différents de la création artistique, des conditions de sa réalisation et de l'attente d'un public, s'est produite à ce moment-là, aboutissant à ce consensus et à ce résultat qui reste mémorable tout au long d'une vie.

\section{Conclusion}

Cette recherche place son centre de gravité dans le spectateur et la trace qu'un spectacle de théâtre a pu laisser en lui, en particulier sa mémoire sonore. Le continuum idéal qui irait de la perception initiale à l'impression et à sa remémoration transmise verbalement, suivant un modèle communicationnel théorique, se révèle très fragile dans la pratique des entretiens. C'est à une véritable recréation que l'on assiste, par les mots, les voix et les sons échangés, retrouvés ou imaginés, tant par l'effort de concentration que par l'association à d'autres souvenirs. Dans cet exercice incertain, le chercheur est entièrement tributaire de la motivation de son interlocuteur, de son envie de témoigner, de transmettre («j’ai plein de documents chez moi », «j'ai vu des spectacles qui peuvent vous intéresser »). En acceptant un entretien portant sur sa mémoire théâtrale personnelle, le spectateur accepte de se mettre en route avec un témoin non familier, dans une quête de souvenirs qui se révèle toujours imprévisible. Plusieurs forces entrainent le moteur de cette démarche de rencontre: l'envie de communiquer des informations factuelles (dates, lieux, répertoire, interprètes, documents), de faire comprendre l'action que le théatre ou certains spectacles ont eue sur soi (par des éléments de chronologie autobiographique), de faire ressentir à autrui ce que l'on perçoit 
comme indicible ou intérieur (par la quête des mots), de faire ressurgir, pour soi et pour l'autre des bribes de théâtre, au-delà de leur évocation verbale (en chantant, par des mimiques du visage, des jeux de mains, des postures). Dans cette volonté de partager le plaisir du théâtre de la manière la plus vive possible, le don de ces mémoires personnelles qui portent sur une longue période et sont recueillies et recréées oralement avec autrui, démontre combien le théâtre, dans toute son auralité, n'est pas qu'éphémère, et accompagne durablement les vies intérieures de ses spectateurs.

\section{NOTES}

1. https://echo-projet.limsi.fr/lib/exe/fetch.php/appel_a_te_moignages_oct._2014.pdf

2. Voir note précédente.

3. Expérience ressentie comme si personnelle qu'elle a donné lieu, à plusieurs occasions, à des suspensions de l'enregistrement, et qu'elle pose aussi la question de la mise à disposition de l'enregistrement ou de sa transcription, même anonymisés.

4. Il est difficile d'éviter des passerelles vers la musique, sa transcription et sa notation, qui, pas plus que les transcriptions verbales, ne sont descriptives et en partie seulement prescriptives. On peut aussi remarquer qu'en musique comme en danse ou en théâtre, on utilise le mot " création » pour désigner la première fois que la partition, la pièce ou la chorégraphie est jouée ou représentée, indiquant le saut considérable ainsi réalisé par cette bascule dans le registre du son et du mouvement dans l'espace.

\section{RÉSUMÉS}

Les entretiens avec des spectateurs, portant sur leur mémoire du théâtre français entre 1950 et 2000 , constituent le terrain et la matière de cette étude. Cette étape de l'analyse interroge autant le processus de remémoration par l'échange verbal que la place de l'expérience auditive initiale dans la constitution d'une mémoire phonique du théâtre, sa pérennité, sa recomposition, son empreinte ou son effacement. Parallèlement aux questions de méthode, quelques pistes sont proposées pour saisir comment les éléments sonores évoqués au long de l'entretien (voix parlée, diction, place du chant et de la musique, ambiance sonore globale, réactions du public) façonnent la mémoire individuelle, comment la quête des souvenirs est associée à leur verbalisation, quelle est la spécificité de la mémoire phonique, et finalement comment le spectateur peut relier explicitement son expérience personnelle à un moment qu'il qualifie d'«historique ", par exemple, une « période Vilar».

Interviewing members of an audience about their memory of French theatre from 1950 to 2000 shapes the ground and matter of this study. This stage of the analysis questions the memorizing process through verbal exchange as well as the place of initial aural experience in the 
constitution of a vocal memory of the theatre, its durability, its recomposition, its imprint or its obliteration. Along with questions of method, some perspectives are proposed concerning the way the sonic elements evoked in the interview (spoken voice, diction, position of song and music, overall sound ambiance, response of the audience) craft the individual memory, the way the quest for recollections is associated with their verbalization, the specificity of vocal memory, at last the way a member of the audience explicitly links his/her personal experience with a moment he/she qualifies as historic, for instance, a « Vilar period ».

\section{INDEX}

Mots-clés : spectateur de théâtre, mémoire théâtrale, mémoire phonique, entretiens

Keywords : audience, vocal memory, theatre memory, interviews

\section{AUTEUR}

\section{HÉLÈNE BOUVIER}

Directeur de recherche au CNRS, anthropologue, HDR en théâtre et arts du spectacle, unité mixte de recherche THALIM « Théorie et histoire des arts et des littératures de la modernité, $\mathrm{XIX}^{\mathrm{e}}-\mathrm{XXI}^{\mathrm{e}}$ siècles », équipe ARIAS « Atelier de recherche sur l'intermédialité et les arts du spectacle », (UMR7172, CNRS, École normale supérieure, Université Sorbonne nouvelle - Paris 3).

Parmi ses publications :

La Matière des émotions. Les arts du temps et du spectacle dans la société madouraise, Paris, École française d'Extrême-Orient, 1994.

Theatre Research International. Special Issue: Anthropology and Theatre (éd. invité), vol. 19, n 1 , Oxford University Press, 1994.

Théâtres d'Asie à l'œuvre. Circulation, expression, politique, avec Gérard Toffin (dir.), Paris, École française d'Extrême-Orient, 2012.

L'Art du pathétique en Asie du Sud-Est insulaire. Le choix des larmes, (dir.), Paris, L'Harmattan, 2013. «Le loddrok. Entre volontés et contraintes, les métamorphoses d'un genre théâtral », Revue d'histoire du théâtre, $\mathrm{n}^{\circ}$ 272, p. 77-144, précédé d'une « Préface : Anthropologie et histoire » de Marie-Madeleine Mervant-Roux, p. 66-76, 2016. 